\title{
Referentes filosóficos del proceso educativo
}

Philosophical references of the educational process

\author{
Oscar-Yecid Aparicio-Gómez ${ }^{1}$ \\ William-Oswaldo Aparicio-Gómez ${ }^{2}$ \\ $E d \& T I C$
}

Recibido: 15.11 .2020

Aceptado: 29.11.2020

\section{Resumen}

Abordar el tema del hombre es de suyo arriesgado, teniendo en cuenta que su dinámica de búsqueda constante limita la reflexión filosófica al campo de los hechos históricos; cabe afirmar también que toda acción humana, tratada con cierto rigor y sistematicidad requiere acudir a la filosofía para indagar, entre otras cosas, por sus fundamentos. Este es el caso del proceso educativo, una realidad que involucra al hombre en sociedad y le ofrece las bases para tomar conciencia de su "ser persona"; así, la antropología filosófica se postula como la reflexión sobre el hombre desde su realidad dinámica e interpersonal de relación consigo mismo y con el entorno que le rodea. Es necesario, pues, buscar el marco argumentativo del hecho y del discurso que constituyen el proceso educativo, labor propia de la filosofía de la educación, y caracterizada como "el saber racional y crítico de las condiciones de posibilidad de la realidad experimental educativa en su conjunto" (Fullat, 1992, pp. 89-90). Desde esta doble perspectiva, se considerarán los fundamentos del proceso educativo.

\footnotetext{
1 oaparicio@editic.net

https://orcid.org/0000-0003-3535-6288

2 waparicio@editic.net

https://orcid.org/0000-0002-8178-1253
}

Volumen 1. Número 2. Julio - Diciembre 2021 


\title{
Palabras clave
}

Pedagogía, Ciencias de la Educación, Familia.

\begin{abstract}
Addressing the subject of man is in itself risky, considering that its constant search dynamic limits philosophical reflection to the factual field of historical facts; It is also possible to affirm that all human action, treated with certain rigor and systematicity, requires turning to philosophy to investigate, among other things, its foundations. This is the case of the educational process, a reality that involves man in society and offers him the basis to become aware of his "being a person"; thus, philosophical anthropology is postulated as the reflection on man from his dynamic and interpersonal reality of relationship with himself and with the environment that surrounds him. It is necessary, therefore, to look for the argumentative framework of the fact and of the discourse that constitute the educational process, a work of the philosophy of education, this is characterized as the rational and critical knowledge of the conditions of possibility of the educational experimental reality in its set. From this double perspective, namely, the very fact of education, and the reflection that bases, guides and projects that same fact, that is, pedagogy, the foundations of the educational process will be considered.
\end{abstract}

Keywords: Pedagogy, Educational Sciences, Family.

\section{Referentes filosóficos de la educación y la pedagogía}

La filosofía cumple un papel indispensable a la hora de dar consistencia al cúmulo de saberes en los que el hombre, a lo largo de la historia, se ha ido configurando como tal. Comencemos por diferenciar los términos referentes al proceso educativo. En primer lugar, a la educación o "hecho educativo" corresponden los procesos socio-históricos y psicosomáticos individuales del educando en relación con su entorno educativo, pero al esfuerzo científico 
por consolidar la acción educativa desde las consideraciones y estudios diversos sobre la realidad, lo que llamamos el discurso, recibe la denominación de pedagogía.

El "hecho educativo" precede a la acción pedagógica; por esta razón es conveniente establecer el soporte antropológico de aquel para luego acompañarlo de una adecuada reflexión pedagógica. Acercarnos a la educación nos pone en contacto directo con una antropología filosófica determinada que nos presenta el tipo de hombre que queremos educar a partir de una adecuada acción educativa basada en la reflexión pedagógica.

\subsection{La educación como hecho educativo}

La educación ha estado siempre ligada al hombre, ella le vincula en su entorno histórico, geográfico y social. Cada cultura propone los derroteros educativos que irá asumiendo cada persona; así, las diversas interpretaciones del mundo y del hombre variarán tanto como puntos de vista culturales existen, lo mismo podría decirse desde el plano subjetivo, cuando se trata de interpretar la realidad.

Esta tensión entre cultura e individuo en lo que atañe a la educación, es presentada por Platón al principio del libro VII de la República, y constituye en sí misma el fundamento -por antigüedad y por validez- de la reflexión educativa. Allí describe en el mito de la caverna una alegoría para explicar la esencia del proceso educativo. Se trata del primer intento de la filosofía y de la educación por sistematizar el sentido último de la paideia griega, refiriéndose con ella simultáneamente a la cultura, a la estética, a la moral y a la política.

Interpretando el mito de la caverna antropológicamente, el ascenso hacia la luz significa el desvelamiento de mi propio ser. Educar sería, pues, un des-ocultar mi ente, mi estructura ontológica fundamental. [...] Así, pues, cabe ya la posibilidad de entender la educación, no como una "actividad social', como una "adaptación a las costumbres de la sociedad", sino a modo de "actividad ontológica" o "antropológica. (Fullat, \& Mélich, 1989, p. 36) 
Como se decía al principio, la educación, la paideia, está íntimamente ligada con la alétheia, la verdad, el des-velamiento. Lo oculto, siguiendo el mito de la caverna, es el ser mismo, lo que el yo realmente es. El proceso para llegar a alcanzar la verdad, por la educación, se hace proyecto, y cada hombre se lanza -en palabras de Sócrates- a la aventura de conocerse a sí mismo para alcanzar la verdad.

\subsection{La pedagogía como discurso educativo}

El ser humano constituye el pilar desde el cual se construye todo proceso educativo, constatable por la experiencia, y circunscrito a una realidad cultural, que requiere un tratamiento más riguroso. Es aquí donde emerge la pedagogía como el esfuerzo científico por consolidar la acción educativa desde las consideraciones y estudios diversos que harán posible descifrar los códigos y procesos culturales para llevar a cabo esta delicada labor de educar.

El fundamento de la pedagogía coincide con la motivación científica por medio de la cual el hecho educativo se hace empíricamente consistente tanto en su método como en sus contenidos. La pedagogía recoge en su haber los aportes que las diversas ciencias pueden brindar para establecer los actores y procesos que hacen posible el hecho educativo.

Ahora bien, no es posible llevar a cabo una acción pedagógica sin una clara idea previa de la naturaleza humana; desde esta perspectiva, la filosofía aparece como encargada de brindarnos un concepto del hombre y su fin. La pedagogía ha de ser, como aparece en la obra de Agazzi:

Una ciencia necesariamente filosófica: es una filosofía aplicada al desarrollo humano en orden a los fines del hombre. [...] La pedagogía, a la luz de la ciencia y la filosofía, aborda el problema de la educación con perfecta conciencia de las necesidades y del progreso del espíritu y de la naturaleza humana: por la pedagogía se convierte la educación en una obra consciente. (Agazzi, 1971, p. 83) 


\section{Persona y educación}

La antropología filosófica parte de una reflexión sobre el hombre, toda ella enmarcada dentro de su compleja realidad personal y social, y determinada sólo en parte por su realidad histórico-geográfica y socio-cultural en la que se haya involucrado, a este respecto puede aparecer iluminadora esta cita de compartida por dos pedagogos catalanes:

El anthropos -hombre- sólo está parcialmente definido por la naturaleza, tanto si se le considera como especie o como individuo; solamente en una dimensión aparece, pues, como dato, como seguridad. La otra cara humana es ser tarea y autorrealización; es preocuparse de lo que puede y debe hacer de sí. Desde lo fijo, lo seguro, el hombre se aventura y vive en la interperie existencial. El ser humano es naturaleza físicopsíquico-social abierta al devenir y a lo posible, lo cual nace de la libertad de elección. (Fullat, \& Mélich, 1989, pp. 93-94)

El hombre es dinamicidad, se mueve en torno a proyectos de futuro, pero con referencia a realidades que ya ha vivido. Es un ser determinado, pero a la vez proyectado; el uso de la libertad como consecuencia de la autodeterminación abre la perspectiva de futuro y adquiere un horizonte de posibilidades para alcanzar los fines propios de su condición humana.

Como se ha explicado antes, la filosofía pretende alcanzar la verdad, que es un proceso, una acción constante por desvelar el ser, un esfuerzo ontológico por buscar lo que se es en sí mismo. La antropología filosófica centra su reflexión en el hombre que se busca a sí mismo, pero no lo hace al azar, sigue unos derroteros que, o bien descubre en su interior, o bien le son dados desde fuera. En la constitución misma del hombre hay una serie de elementos que le determinan -"pathos" o lo natural dado- y otros que le proyectan desde la realidad propia al mundo circundante -“ethos” o lo social recibido-; así, el hombre está ontológicamente traspasado por la dinamicidad, manifestada en su desarrollo biológico y psicológico, pero también socio-cultural. En este contexto aporta ideas la afirmación de Fullat y Mèlich, según la cual "el hombre se experimenta a la vez como dato y como quehacer, como determinación 
y como atrevimiento, como realidad y como posibilidad” (Fullat, \& Mélich, 1989, pp. 93-94). El soporte antropológico que aporta la filosofía hace posible la comprensión amplia, por su carácter ontológico, de la concepción de hombre, situado en la realidad cambiante y a la vez maleable, perfeccionable desde su propia condición.

“[...] El hombre en proceso de educación establece el objeto propio de la antropología filosófica de la educación; el acto educante posibilita la comprensión del ser humano, mientras los paradigmas existenciales antropológicos nos hacen comprensible el acto educante" (Fullat, 1992, p. 107). Urge ayudar a cada hombre para que pueda descubrir en sí mismo su estructura ontológica, lo que él es. Ahí es donde la educación hace posible que todas las potencialidades del hombre puedan ser actualizadas en un contexto y en una cultura determinadas; su labor educadora y responsabilidad es de tal magnitud que configura a lo largo de tiempo el modelo de hombre, de cultura y de historia.

\subsection{La persona, que se sabe y se hace a sí misma}

La reflexión nos trae en este punto a considerar que el ser humano participa de una naturaleza físico-psíquico-social abierta al devenir y a lo posible. La apertura a su propia condición sólo puede ser viable cuando el hombre hace un ejercicio libre de su voluntad, esto es, acompañar el proceso de manifestación de su propia conciencia, a la vez que se deja orientar por quienes ya han participado del proceso. De ahí que es necesario sentar las bases de una pedagogía de la afirmación de sí mismo sobre la conciencia personal y comunitaria, como camino para preparar el nacimiento de la libertad de elección, ejercida con autonomía.

E. Stein afirma que "en la naturaleza de un hombre está prevista su llamada, su vocación y profesión, es decir, la actividad, el trabajo hacia el que está orientado desde lo profundo" (Torralba, 2000, p. 133). El uso del término persona, es decir, el núcleo ontológico del individuo que tiene conciencia de sí mismo, se autodetermina libremente y es responsable, es el más adecuado para hablar del hombre desde la perspectiva individual pero a la vez relacional; el hombre no es sólo una realidad individual, sus diversas manifestaciones 
constituyen un mundo lleno de realidades suficientes como para dirigirse a él mismo y a los otros con la conciencia de que junto a sus situaciones límite está determinado también por su libertad, su lenguaje, etc. En este contexto puede ser complementaria la afirmación de que "todo individuo humano, en sentido ontológico y ético (personalidad), cualquiera que sea su individualidad antropo-psicológica, ha de ser considerado y tratado como un valor en sí y nunca será lícito supeditarles a objetivos extraños de sí mismo” (Göttler, 1965, p. 121).

Desde este modelo de "persona", quedan sentadas las bases del fin de la educación: acompañar a cada hombre en su proceso de personalización. Las potencialidades humanas (dinamicidad, proyección, libertad, etc.) están ahí para ser dirigidas mediante un adecuado proceso educativo, orientado desde una perspectiva pedagógica ajustada a cada cultura, hacia la realización personal, es decir, a la personalización de cada hombre.

El trasfondo personalista -que se irá desarrollando en los diversos apartados-, entendido como la afirmación del primado de la persona humana sobre lo que no es la persona humana, será el modelo antropológico latente durante esta exposición. Para acercarse a las consideraciones personalistas parece iluminador el siguiente texto: "Una cosa es lo que yo soy y otra cosa muy distinta es aquel que soy; lo primero es mi qué, mi "naturaleza", y lo segundo es mi quién, "mi persona". [...] En la entraña del ser "persona" se descubre la libertad. [...] Los hombres, personalizados, tejen entre ellos relaciones libres, conscientes y respetables" (Fullat, 1992, p. 302). Esta perspectiva abre a la persona un horizonte hacia las relaciones humanas. La proyección que tiene el hombre hacia los otros le hará encontrar en ellos los derroteros que le marquen cómo alcanzar su autorrealización. Cada hombre por su propio esfuerzo se hace consciente de sus limitaciones, una de ellas es la no poder realizarse solo, es ahí donde encuentra en el otro el sentido de su vida. La educación aparece necesaria desde una adecuada consideración pedagógica para que el hombre no considere al otro como un medio para su realización, sino como un fin en sí mismo, un complemento necesario en su realización personal. 


\subsection{La persona, que se sabe y se hace siendo con los otros}

Hasta aquí tenemos que cada persona debe recorrer el camino de la vida bajo su propia responsabilidad, pero en determinados procesos necesita la guía de otras personas que ya han recorrido un camino similar. El contacto interpersonal hace posible que cada hombre llegue a expresarse y a hacerse comprensible, especialmente a partir de las relaciones sociales, marcadas todas ellas por el influjo educativo que encuentra a su alrededor.

El hecho de presentar a un hombre inacabado no sugiere sólo la necesidad de actualizar las potencialidades que trae consigo en el ámbito individual, representa además la motivación que hace posible llevarlas a plenitud; es aquí donde la apertura al otro desde la conciencia del yo constituye el camino de perfección personal. Puede deducirse que la persona a partir de sus determinaciones esenciales genera cultura, "determinada por un conjunto de moldes, de conocimientos y de conducta, que pasa a los educandos gracias a la habilidad de los educadores, los cuales se hallan inscritos en las diversas instituciones sociales, por ejemplo, en la familia y en la escuela" (Fullat, 1992, p. 185).

El hombre hace manifiesta la cultura y el proceso educativo hace posible perpetuarla. Aquellos que ya conocen los procesos y desarrollos del saber sirven de guía a quienes todavía no los poseen. De ahí que el interés primordial del educador es el bien de su educando y por eso debe velar por su vida y por el sentido de su vida. [...] El educador no sólo tiene que dejar vivir al educando, no es suficiente con dejarle hace su vida, debe ayudarle a dar sentido a su vida, a vivirla con intensidad, con pasión” (Torralba, 2000, p. 177). La comunicación linguística de señales y significados entre educador y educando es fundamental para integrar la personalidad de este y señalar los derroteros sociales que dan contexto al desarrollo de su personalidad. Codificar y descodificar sucesivamente los códigos lingüísticos hacen posible que dicha relación se haga comprensible y pueda dirigirse hacia un punto común, la realización personal del educando. 
Dando un paso adelante, y haciendo resonar una tesis de Pestalozzi, tenemos que:

Solamente de la familia puede surgir la sociedad auténtica y popular. Por eso cada familia debería ser una escuela y cada escuela una familia. [...] Con su espíritu sencillo e imperturbable, con su perseverancia longánime en el trabajo cotidiano, con su paciencia en soportar al prójimo y conducirlo a sus fines a pesar de todas las dificultades, la familia inculca la firmeza, la uniformidad e idealidad del carácter, da la base para la formación del hombre. (Agazzi, 1971, pp. 55-56)

La familia encarna al primer educador y constituye el primer agente socializador y orientador de la vida de cada persona; depende de ella que las motivaciones humanas adquieran un horizonte consistente de sentido y de realización personal. La familia es el lugar donde cada persona aprende a saberse, es decir, a tener conciencia de sí mismo como artífice de su propia historia, constituyen el marco experiencial de personalización de cada hombre, le ofrece lo medios para desarrollar lo que ya es, ayudándole a tomar conciencia de sí mismo mediante la autenticidad de las relaciones, comportamientos y actitudes. El camino de la vida en compañía de otros hace madurar la vocación de cada uno y a la vez puede hacerla comprensible para quienes le rodean. El que una persona haya encontrado su puesto en la vida hace posible que otros tengan un prototipo, un eje orientador que le pueda acompañar mediante un adecuado proceso educativo, y marcar así los derroteros propios de quien busca su realización personal.

\section{Teleología del proceso educativo}

El lugar propio de la educación ha ido apareciendo circunscrito al campo de la relación: se educa para hacer posible el desarrollo de las potencialidades del hombre, pero a la vez se educa para que cada hombre, una vez alcanzada la madurez que le es propia, pueda convertirse en agente educativo y crear así una dialéctica que actualice la creación de cultura basada en las relaciones interpersonales. 
La persona, formada, educada y socializada, encuentra que su propia dinamicidad "irrumpe con tal ímpetu que no tolera ni perspectivas unilaterales ni horizontes limitados. La dialéctica aparece entonces y esclarece el perfil de la persona. Tal dialéctica revienta la inmanencia de la persona a la Trascendencia divina; la persona humana se reconoce en aquel instante imagen de Dios, el cual es pura gratuidad y no el resultado de discurso o de deseo" (Fullat, 1992, p. 302). Más allá del discurso, la realidad trascendente aparece como el ideal hacia el cual tiende la persona que se hace consciente de su finitud, de su limitación y de su provisionalidad, expresada en la conciencia personal de estar circunscrita en un constante proceso educativo que no se puede considerar como totalmente finiquitado.

Afirmar que un educando es "persona" desliga la consideración del hombre al mero saber científico, es más, desde esta perspectiva cobra sentido la referencia al trascendente; las relaciones interpersonales, en especial las que vinculan a la persona dentro del contexto familiar o escolar, tienden a una perfección que no se agota en el misterio relacional humano. La motivación última de la educación, es decir, el amor a los educandos, es el motor que hace posible llevar a cabo el proceso educativo hasta los límites de la humanidad y desentrañar su sentido último, aparece así el Misterio del Trascendente como proyección del hombre hacia el fin que constituirá su ser persona en plenitud.

[...] Ni el amor a los educandos en el acto pedagógico, ni el amor a los conciudadanos en los actos social y político llega jamás a su plenitud. [...] El amor puede entenderse como un eco de lo Absoluto -"Dios creó al hombre a su imagen: lo creó varón y mujer" (Gn 1, 27)-. El Absoluto no se desentiende de cuanto ha lanzado amorosamente a la existencia -"Dios ama todo cuanto existe y no abomina nada de lo que hizo" (Sab 11, 24). [...] El hombre "es", pero carece de consistencia; por eso la busca procurando alcanzar su propia identidad. La amenaza de la muerte y de la nada sólo podemos vencerla, según E. Bloch, esperando en un más allá de lo que somos. Con estas perspectivas, la educación penetra en una dialéctica "historia-eternidad", que le confiere sentido y le inyecta entusiasmo. (Fullat, 1992, p. 302) 
Y es esta misma dialéctica la que mueve a cada hombre hacia una búsqueda de sentido. Si la pedagogía se desentiende de la proyección dinámica del hombre hacia el Misterio de su propia trascendencia ignorará también que el fin del proceso educativo tenga su razón de ser precisamente en su origen, llevar al hombre a ser lo que realmente es. Una antropología entendida desde la perspectiva personalista, es decir, que considera al hombre como un fin en sí mismo, no puede desentenderse de la referencia a Dios, porque en Él se fundamenta toda acción educativa y cobra sentido cualquier reflexión pedagógica.

\section{Conclusiones}

La educación constituye un elemento integrador de la sociedad, por medio de ella el hombre puede desarrollar todas sus potencialidades y llegar a ser lo que, en efecto, está en capacidades de lograr. Sin embargo, debe tenerse en cuenta que para educar hacen falta medios que estimulen el proceso, la pedagogía orienta este esfuerzo que sólo será efectivo si se define de entrada el tipo de hombre que se quiere formar.

Teniendo en cuenta que el hombre está configurado por la dinamicidad que le hace moverse en torno a proyectos de futuro con referencia a realidades que ya ha vivido, la educación hace posible que todas las expectativas humanas puedan ser actualizadas en un contexto y en una cultura determinadas. Parece acertado proponer el criterio de persona para entender desde ella al hombre que se quiere formar, es decir, un ser espiritual guiado por una escala de valores y en constante proceso de realización; con este supuesto puede iniciarse, pues, el acompañamiento humano en el proceso de personalización, formación o educación.

Un elemento que resulta definitivo a la hora de establecer los supuestos del proceso educativo es el de las relaciones interpersonales, las cuales hacen posible que el hombre pueda expresarse y a hacerse comprensible, tanto para transmitir experiencias como para hacerse partícipe de ellas. En este contexto social la familia aparece como el lugar donde cada persona aprende tener conciencia de sí mismo como artífice de su propia historia mediante el proceso de personalización en el que cada ser humano se ve envuelto. 
La motivación última de quien enseña consiste en sentirse realizado enseñando el camino de la realización a otros, el amor, por tanto, es el motor que mueve esta relación; de ahí que todos los medios pedagógicos deben conducir a este fin, nada más claro, en este sentido, que la presencia del Misterio del Trascendente como configuración última de ese fin, la plenitud de la realización personal que posee ya en esa condición un carácter de definitividad.

\section{Referencias}

Agazzi, A. (1971). Historia de la filosofía y la pedagogía I. Alcoy: Marfil.

Aparicio-Gómez, Jaime-Orlando (2016). El diseño tecnopedagógico en la educación básica primaria, secundaria y media. En Aparicio-Gómez, Oscar-Yecid (Eds.). El Uso Educativo de las TIC (pp. 11-23). Bogotá, Colombia: Universidad Central. ISBN: 978-958-26-0316-8

Aparicio-Gómez, Oscar-Yecid (2017). Uso de las TIC en el Colegio Padre Manyanet - Chía, Colombia (2011-2014). En Aparicio-Gómez, Oscar-Yecid y Aparicio-Gómez, WilliamOswaldo (Eds.). Uso de las TIC para la innovación (pp. 129-158). Bogotá, Colombia: Ed\&TIC. ISBN: 978-958-26-0367-0.

Aparicio-Gómez, Oscar-Yecid, Aparicio-Gómez, William-Oswaldo y Hernández, J. (2020). Formación del profesorado en ambientes virtuales de aprendizaje. En: Hinojo, F., et. al. (Eds.). Experiencias e Investigaciones en Contextos Educativos (pp. 604-613). Madrid: Dykinson S.L. ISBN: 978-84-1377-171-7.

Fullat, O. (1992). Paideia, filosofías de la educación. Barcelona: Paideia - CEAC.

Fullat, O. \& Mélich J. (1989). El atardecer del bien, Bellaterra: UAB.

Göttler, J. (1965). Pedagogía sistemática, Barcelona: Herder:

Torralba, F (2000). Explorar el sentido de la realidad, Barcelona: Edebé. 